\title{
Imaging Three-Dimensional Heliosphere in EUV
}

\author{
Mike Gruntman ${ }^{1}$, Mike Lampton $^{2}$, Jerry Edelstein ${ }^{2}$ \\ ${ }^{1}$ Astronautics and Space Technology Division, Viterbi School of Engineering, \\ University of Southern California, Los Angeles, CA 90089-1192; mikeg@usc.edu \\ ${ }^{2}$ Space Sciences Laboratory, University of California, Berkeley, CA 94720
}

\begin{abstract}
The glow of interstellar plasma and solar wind pickup ions and solar wind emissions at $30.4 \mathrm{~nm}$ provide a way of exploring important physical processes in the heliosphere. Imaging the heliosphere at this wavelength with high spectral resolution will map the heliopause, probe pickup ions in the solar wind, and reveal the three-dimensional flow pattern of the solar wind, including in the regions over the sun's poles. The required high-throughput, high-resolution spectrometer for diffuse radiation should be able to measure 1 milli-Rayleigh irradiance in 10000 seconds with a $0.005-\mathrm{nm}$ spectral resolution across pixels subtending a few degrees of celestial arc. The desired performance characteristics can be achieved by combining multiple entrance slits with an optimized spectrometer design. We present a concept of a space experiment to image the heliosphere at $30.4 \mathrm{~nm}$ and discuss the scientific rationale and required instrumentation.
\end{abstract}

Keywords: heliosphere, heliopause, extreme ultraviolet, EUV, solar wind, diffuse radiation, spectrometry

\section{SOLAR SYSYTEM GALACTIC FRONTIER}

\subsection{Global interaction}

The interaction of the expanding solar wind plasma with the surrounding galactic medium - Local Interstellar Medium (LISM) - creates the heliosphere. ${ }^{1-4}$ The heliosphere is a complex phenomenon where the solar wind and interstellar plasmas, interstellar gas, magnetic field, and energetic particles all play important roles. ${ }^{5-8}$ The region where the solar wind meets the galactic medium remains poorly explored, with the present concepts of the heliospheric interface based on scarce and mostly indirect experimental data. The most distant operating spacecraft, Voyager 1, had reached the termination shock region and the spacecraft entered the slowed down solar wind at $94 \mathrm{AU}$ (ecliptic latitude $\beta=34.8^{\circ}$ and ecliptic longitude $\lambda=253.3^{\circ}$ ) in December 2004. (The first inconclusive indications of the possible arrival at this region were reported two years ago. ${ }^{9,10}$ ) This is a major milestone in exploration of the outer heliosphere, although the spacecraft is instrumented for planetary flybys and not optimized for interstellar studies. Voyager 1 will be turned off in 2020-2025 at the distance $150 \mathrm{AU}$ because of the decreasing efficiency of its radioisotope thermoelectric generators.

A possible two-shock Sun-LISM interaction scenario ${ }^{5}$ illustrates the main features of the heliosphere (Fig. 1). The interstellar wind approaches the heliosphere with a supersonic velocity and forms a bow shock. The dynamic pressure of the expanding, highly supersonic solar wind decreases with the heliocentric distance. At a certain distance from the Sun, this pressure would equal the external LISM pressure of the interstellar wind and magnetic field. The solar wind expansion transitions to a subsonic flow at the termination shock. There the kinetic energy of the supersonic flow is largely converted into thermal energy in the subsonic plasma beyond the shock. The subsonic postshock solar wind plasma (Fig. 1, left) flows in the heliospheric sheath around the termination shock and down the heliospheric tail, where it eventually mixes with the interstellar galactic plasma at distances $>5000 \mathrm{AU} .^{11}$ The galactic plasma (Fig. 1, right) flows around the heliopause, the boundary separating solar and galactic plasmas; interstellar neutral atoms penetrate the heliosphere, some reaching the Sun's vicinity at 1 AU.

Our present experimental capabilities to explore in-situ the solar system frontier are limited by the technical difficulties and budgetary realities of sending space probes to this distant region and beyond. At the same time, the structure of and the physical processes at the solar system frontier - the heliospheric interface - are of fundamental importance for un-

Solar Physics and Space Weather Instrumentation, edited by Proceedings of SPIE Vol. OEI133 (SPIE, Bellingham, WA, 2005) - 0277-786X/05/\$15 - doi: 10.1117/12.614492 


\section{ENA Imaging of the Heliosphere}

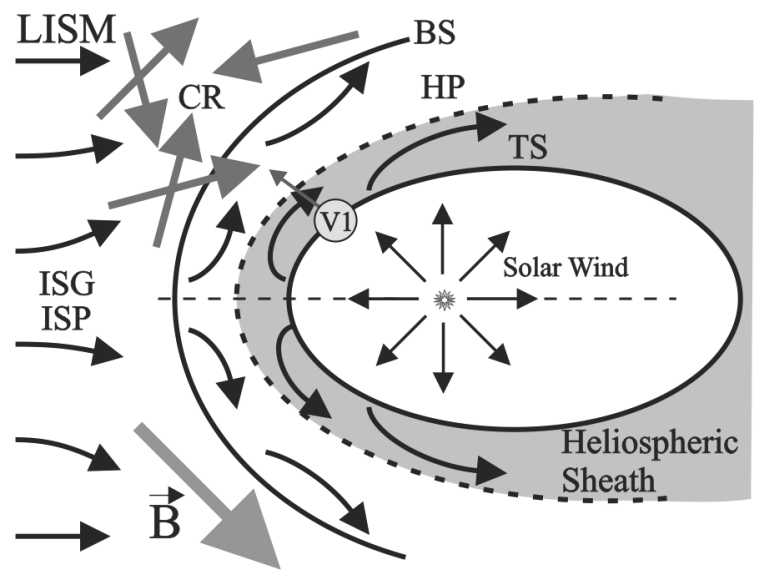

EUV Mapping of the Heliopause

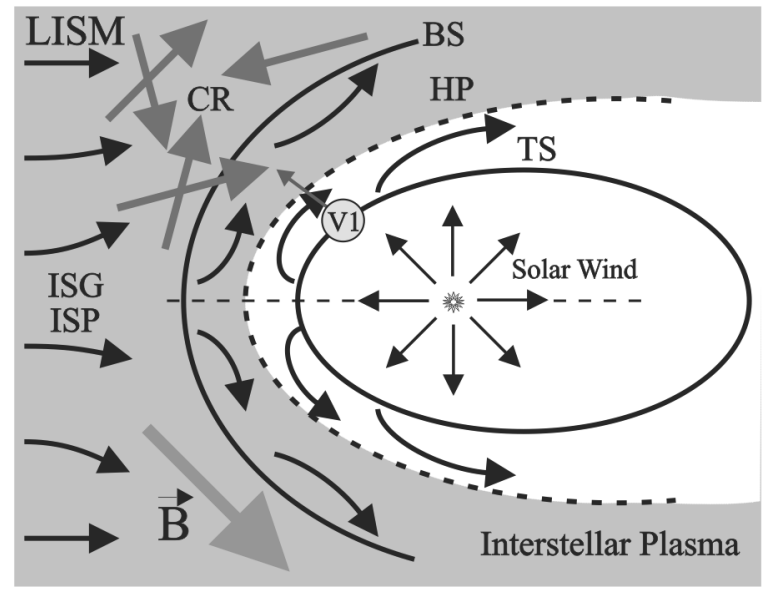

Figure 1. Concept of the interaction of the solar wind with the local interstellar medium. LISM - local interstellar medium; TS termination shock; HP - heliopause; BS - bow shock; CR - cosmic rays; ISP(G) - interstellar plasma (gas); B - magnetic field. The circle V1 shows the position of the Voyager 1 spacecraft entering the heliosheath - the region between the termination shock and the heliopause - in December 2004. Left: the heliospheric sheath (gray) contains the postshock subsonic solar wind plasma and pickup protons. The ions produce energetic neutral atoms (ENA) and the heliospheric sheath will be remotely probed by imaging in ENA fluxes ${ }^{12}$ by the Interstellar Boundary Explorer (IBEX) mission. ${ }^{13}$ Right: interstellar plasma (gray) flows around the heliopause; the heliopause will be mapped and the plasma flow beyond probed by imaging in EUV, as discussed in this article.

derstanding the interaction of our star, the Sun, with the galactic medium. This region also needs to be charted for optimizing our first foray into interstellar space by the Interstellar Probe mission and for supporting the truly interstellar exploration and interstellar travel of the future. The study of the outer heliosphere and its interaction with the galaxy will remain a top NASA priority, a kind of exploration that NASA does best.

\subsection{Asymmetric heliosphere}

Three fundamental factors make the heliosphere essentially asymmetric: (1) the motion of the Sun with respect to the surrounding interstellar medium, sometimes described as the interstellar wind; (2) asymmetry of the solar wind flow in heliolatitude; and (3) interstellar magnetic field of the unknown magnitude and direction. The interstellar wind velocity and direction are well established: $26 \mathrm{~km} / \mathrm{s}$ (or $5.2 \mathrm{AU} / \mathrm{yr}$ ) and $\beta \approx 5^{\circ}$ and $\lambda \approx 255^{\circ}$ (ecliptic coordinates), respectively. The solar wind flow is highly anisotropic during solar minimum ${ }^{14}$ with the typical velocities near ecliptic $450 \mathrm{~km} / \mathrm{s}$ and over the poles $750 \mathrm{~km} / \mathrm{s}$. (The Sun spin axis is tilted $7.25^{\circ}$ from the normal to the ecliptic plane.) The direction of interstellar magnetic filed in the sun's vicinity is unknown. It is anticipated that both the heliopause and termination shock shapes will reveal the asymmetry of the magnetic field, with the effect being much stronger for the former. (The difference in the derived directions "at infinity" of the interstellar helium atom and hydrogen atom fluxes reaching the inner heliosphere $^{15}$ would also depend in a complex way on the interstellar magnetic field, as was argued for the last 20 years.)

If Voyager 1 is operational when the spacecraft reaches the heliopause, the shapes of the heliosphere and of the heliopause and directional variations of the nature of the heliospheric sheath and processes therein will still remain unknown. Note that the spacecraft probes the essentially asymmetric three-dimensional heliospheric boundary in one point-direction as the future Interstellar Probe will do. Interstellar Probe will go to 300-400 AU in the approximately the same direction as Voyager 1. The heliospheric boundary is believed to be closest in the upwind (with respect to the interstellar wind) direction, making it optimal for sending a spacecraft to interstellar medium. ${ }^{16}$ Clearly, only remote techniques, supported and validated by the "ground-truth" in-situ measurements by Voyager and Interstellar Probe, will enable a comprehensive exploration and "putting on the maps" the solar system galactic frontier. The sheer size of the heliosphere calls for remote techniques to probe its global three-dimensional properties. ${ }^{17-19}$ 


\subsection{Heliosphere imaging}

Several experimental techniques indirectly probed the heliospheric interface region: measurements of interplanetary glow at 121.6 and $58.4 \mathrm{~nm}$, pickup ions in the solar wind, cosmic rays, and anomalous cosmic rays; direct detection of interstellar helium atoms; and spectroscopic observations of nearby stars and interstellar medium. All these physical phenomena either depend in a weak way on the properties of the three-dimensional heliospheric interface or are sensitive to the interface structure in the upwind (interstellar wind) direction only. The available experimental data are indirect and require use of complex modeling, with many fundamental model assumptions not verified experimentally, for inferring the processes at the solar system frontier.

Two new most promising concepts have emerged to remotely sense the heliospheric interface. These concepts rely on emissions originating at the solar system galactic frontier and reaching the observer at $1 \mathrm{AU}$ with little disturbance. First, heliosphere imaging in fluxes of energetic neutral atoms (ENAs) will probe the plasma properties in the heliospheric sheath between the termination shock and the heliopause (Fig 1, left). The concept of ENA imaging of the heliospheric interface originated in early 1980s. ${ }^{20,21}$ Today, the concept ${ }^{12}$ and instrumentation ${ }^{17,22}$ are mature. In January 2005, NASA selected a dedicated mission, Interstellar Boundary Explorer (IBEX), ${ }^{13}$ to perform heliosphere ENA imaging. Global ENA images would clearly differentiate ${ }^{12}$ between physically distinct possibilities of processes at the termination shock and beyond and reveal properties of the plasma in the heliospheric sheath. IBEX will be launched in 2008 into a highly eccentric orbit, with a sun-pointed spinning spacecraft obtaining all-sky ENA images in several energy bins each six months. ${ }^{13}$

The second concept to map the heliopause in extreme ultraviolet (EUV) and to explore the flow of interstellar plasma around it (Fig.1, right) was formulated in 1990s. ${ }^{18,19,23,24}$ The most promising spectral line for such an experiment is the resonance line of a singly-charged helium ion, $\mathrm{He}^{+}, 30.378 \mathrm{~nm}$ (often referred to as the 30.4-nm line). Imaging in EUV would be a logical next step in exploration of the solar system frontier, ${ }^{16,18,19}$ building upon and following heliosphere imaging in ENA fluxes by IBEX. EUV imaging of the global heliosphere requires advancing the instrumentation stateof-the-art by a factor of 100 , with the new instrumental concept formulated and presently being pursued. ${ }^{18,19,25,26}$

\section{HELIOSPHERE IN EXTREME ULTRAVIOLET}

Three main sources contribute to radiation fluxes at 30.4-nm, the spectral band of interest: the glow of interstellar plasma and pickup ions; emissions of the solar wind; and galactic emissions (stars and hot plasmas in the Local Bubble). Measurement of the glow of interstellar plasma opens a way of mapping the heliopause. The pickup ion glow and solar wind emissions present the foreground radiation at this wavelength and galactic emissions are the background.

Two most abundant ions of interest in interstellar plasma are singly charged helium $\mathrm{He}^{+}$and singly charged oxygen $\mathrm{O}^{+}$ ions, with the corresponding spectral lines at 30.4 and $83.4 \mathrm{~nm}$, respectively. (Protons cannot be imaged optically; in contrast, the way to image proton populations is by measuring fluxes of ENAs. ${ }^{17}$ ) Expected radiance of interstellar ion glow would be in the milli-Rayleigh range for helium and the micro-Rayleigh range for oxygen. ${ }^{18,19,23,24}$ ( 1 Rayleigh $=1$ $\mathrm{R}=10^{3} \mathrm{mR}=10^{6} / 4 \pi$ phot $/ \mathrm{cm}^{2} \mathrm{sr} \mathrm{s}$.) Radiance of $1 \mathrm{mR}$ is a flux of one photon per square centimeter per second from a solid angle of $6.4^{\circ} \times 6.4^{\circ}$. Measurement of milli-Rayleigh radiances presents a major instrumental challenge, and recording sky maps in the micro-Rayleigh range is unrealistic. Therefore, we will concentrate on radiation at $\mathrm{He}^{+}$resonance line.

Scattering of solar line photons by ions produces a glow. Therefore the glow is spectrally concentrated within the corresponding solar line. In contrast, emissions are produced by transitions in moving $\mathrm{He}^{+}$ions, with the photons Dopplershifted. Figure 2 shows a typical spectral radiance of the three main radiation sources at $30.4 \mathrm{~nm}$ (see details below). ${ }^{18,19}$

\subsection{Glow of interstellar plasma and pickup ions}

Singly charged helium ions in the Sun's vicinity would scatter the solar 30.4-nm line radiation producing the glow. Two major populations of $\mathrm{He}^{+}$ions would contribute to the glow. First, there are helium ions in the plasma of the local interstellar medium surrounding the solar system. This plasma cannot penetrate the heliosphere and flows around the heliopause (Fig.1; right). The plasma density within the heliopause rapidly decreases with the expansion of the solar wind. Therefore, one can image the sun surrounded by an interstellar plasma "wall" beyond an empty cavity, the 


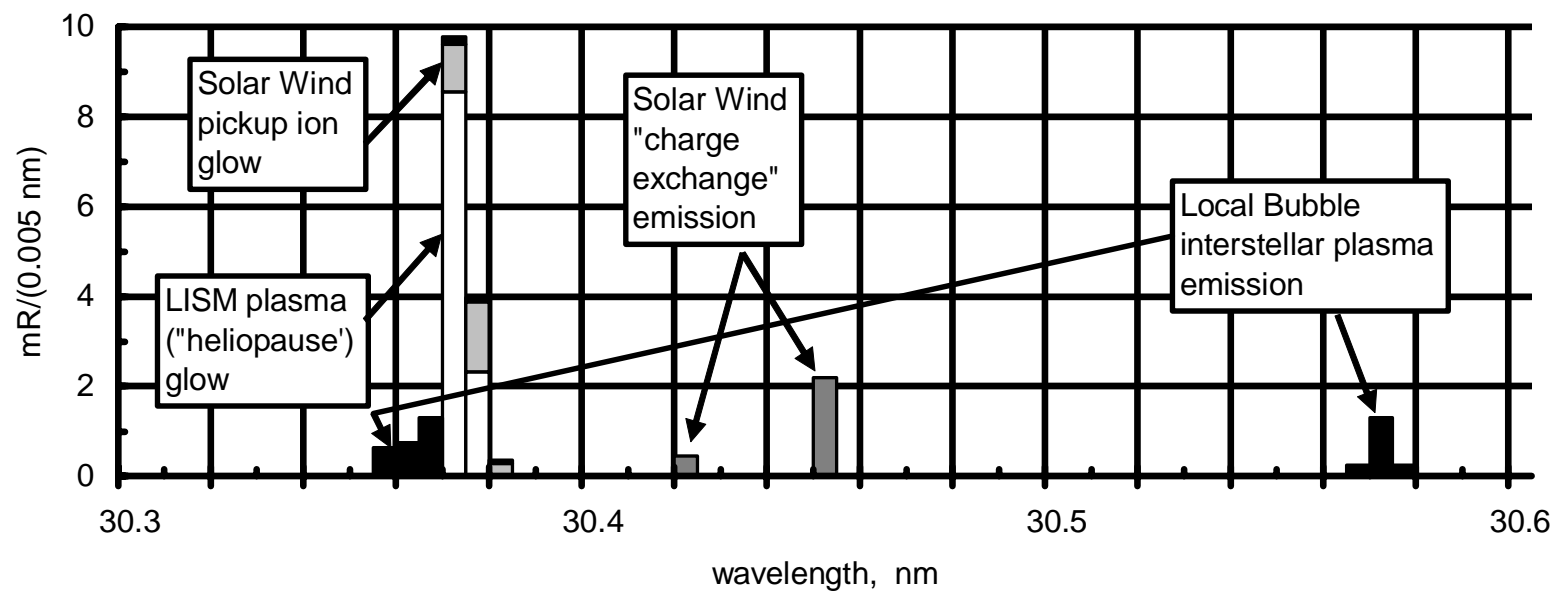

Figure 2. Representative spectral radiance (at $1 \mathrm{AU}$ ) at $30.4 \mathrm{~nm}$ summed over $0.005-\mathrm{nm}$ bins for an observational direction partially in the slow and partially in the fast solar wind. ${ }^{18,19}$ The glow of the LISM plasma beyond the heliopause is shown as white (empty) bars. Also shown are the pickup ion glow (light gray); Local Bubble emissions (black); and the Dopplershifted solar wind charge-exchange emissions (dark gray; the two peaks correspond to the fast polar solar wind and slow ecliptic solar wind).

"heliopause moat," limited by the heliopause boundary. ${ }^{18,19}$ We do no know the distance to the heliopause, its shape, and whether it is stable. The estimates suggest that the heliopause could be somewhere between 140 and $180 \mathrm{AU}$ in the upwind direction and farther away in other directions. For a simplified case of a vanishing ion number density inside the heliopause and for interstellar plasma at rest and with a uniform number density and temperature beyond the heliopause, the expected radiance of the interstellar plasma glow is inversely proportional to the distance to the heliopause. ${ }^{19,23,24}$ The measurement of the interstellar plasma glow would thus open a way to map the heliopause and probe the flow properties of the interstellar plasma beyond.

The second ion population contributing to the glow is singly charged helium pickup ions in the solar wind. Interstellar helium atoms are ionized in interplanetary space and picked up by the solar wind plasma flow. The origin of pickup ions is well understood and their properties have been extensively studied experimentally and theoretically. ${ }^{27-29}$

Figure 3(d) shows the calculated all-sky map (ecliptic coordinates) of the glow of the interstellar plasma and solar wind pickup ions at $30.4 \mathrm{~nm} .{ }^{18,19}$ The map exhibits two maxima, one broad enhancement in the upwind direction (ecliptic longitude $240-270^{\circ}$ ) and the other narrower peak in the exactly opposite downwind direction. The glows of the interstellar plasma and pickup ions show essentially different directional dependence with the upwind radiance dominated by the interstellar plasma and the downwind peak due to the pickup ions. Note that the interstellar plasma glow is proportional to the ionized component of interstellar helium, while the pickup ion glow is proportional to the neutral component of interstellar helium. ${ }^{19}$ Therefore, one can establish the ionization state (in the LISM) of interstellar helium by measuring the upwind-to-downwind ratio of radiance at $30.4 \mathrm{~nm}$. The all-sky radiance (brightness) distribution will map the heliopause and probe the flow pattern of the interstellar plasma beyond. The asymmetry of the interstellar magnetic field vector would also prominently manifest in the sky map.

\subsection{Solar wind emissions}

Charge-exchange collisions between the solar wind alpha particles $\left(\mathrm{He}^{2+}\right.$ ions) and heliospheric atomic hydrogen would produce unique emissions at $30.4 \mathrm{~nm} .{ }^{18}$ Helium is the second most abundant component of the solar wind, with alpha particles typically constituting $4-5 \%$ of the number of plasma ions. Interstellar hydrogen atoms, the most abundant component of the neutral gas in the LISM, penetrate and fill the heliosphere, providing the background gas of atomic hydrogen for charge-exchange collisions. In addition, heliospheric ENAs could significantly contribute (and even dominate under some conditions) neutral hydrogen number densities in the sun's vicinity. ${ }^{30}$ 
Charge exchange collisions between alpha particles and hydrogen atoms produce protons and singly ionized helium ions. The ionization potential of a hydrogen atom is nearly equal to that of a $\mathrm{He}^{+}$ion in the first excited state. Therefore, the most efficient branch of charge-exchange collisions would be accidentally resonant charge exchange transfer producing helium ions in the first excited state $\mathrm{He}^{+}(2 \mathrm{p})$ and $\mathrm{He}^{+}(2 \mathrm{~s})$. The $\mathrm{He}^{+}(2 \mathrm{p})$ ions would quickly $(0.1 \mathrm{~ns})$ decay to the ground state with the emission of a 30.378-nm photon. The efficiency of such charge exchange strongly depends on the collision velocity, with the corresponding cross section being 3.3 times higher for typical velocities of the fast (polar) solar wind than for typical velocities of the slow (ecliptic) solar wind. ${ }^{18}$

\section{(d)}
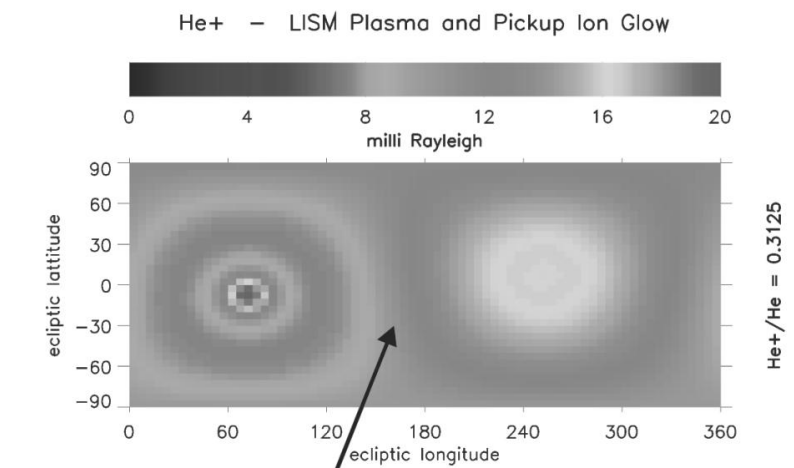

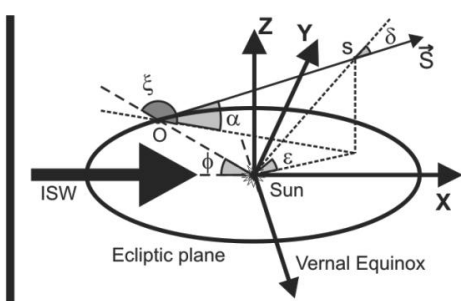

(c)

ISW - interstellar wind $(\sim 26 \mathrm{~km} / \mathrm{s})$; assumed to be in the ecliptic plane.

S - observation direction vector

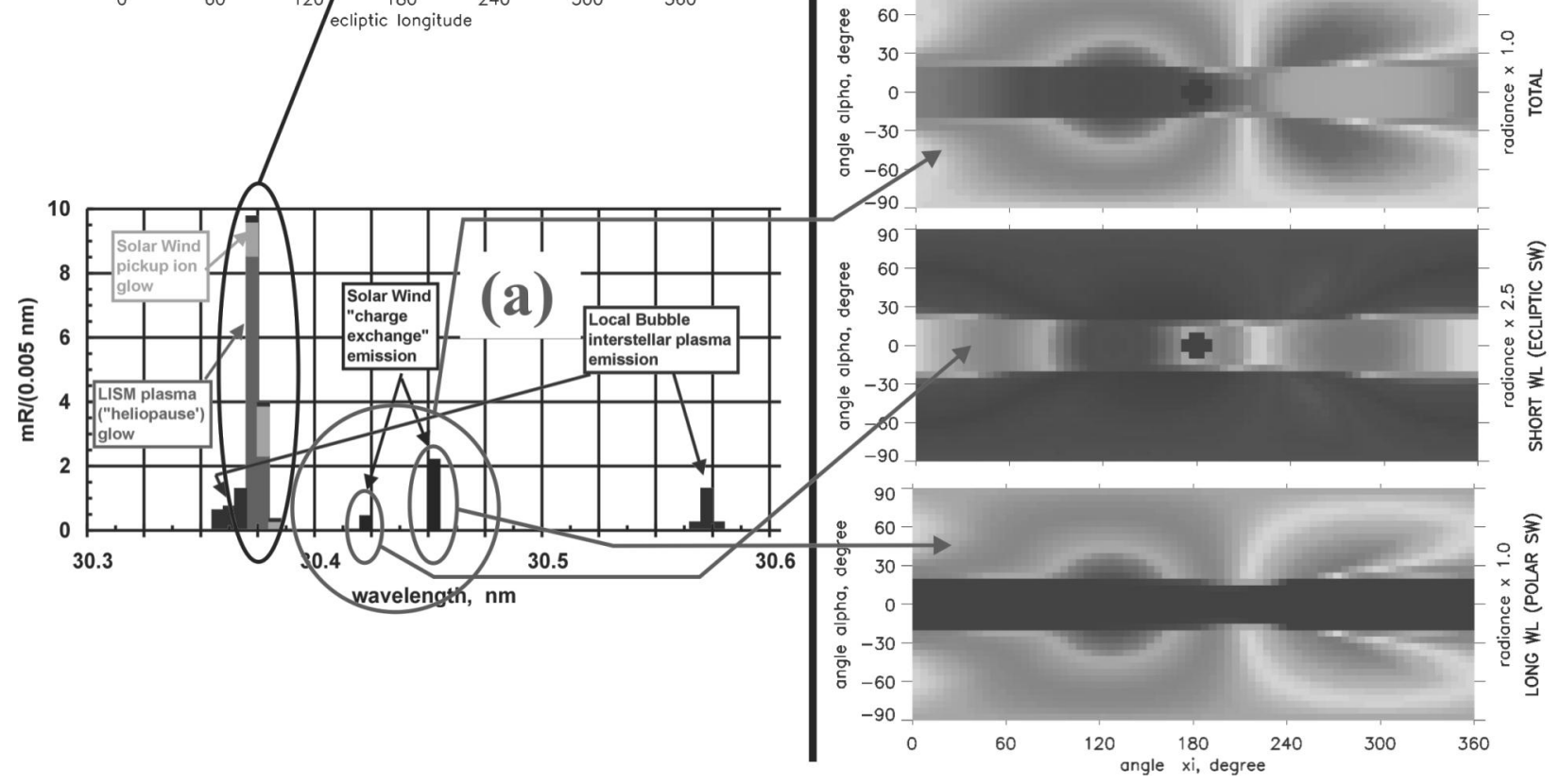

Figure 3. (a) - Representative spectral radiance (at $1 \mathrm{AU}$ ) summed over 0.005 -nm bins for an observational direction partially in the slow and partially in the fast solar wind. The solar wind emission (black bars) shows two distinct Doppler-shifted peaks, produced by charge exchange in the slow and fast flows, respectively. Dark-gray bars are the glow of the LISM plasma beyond the heliopause "heliopause glow"). Light-gray bars are the glow of helium pickup ions in the solar wind. Other black bars, as marked, are the line emissions of hot plasma in the Local Bubble. (b) - All-sky images in the solar wind 30.4-nm emission. Top: total red-shifted solar wind emissions. Sky maps in the spectral ranges corresponding to the $250-500 \mathrm{~km} / \mathrm{s}$ ("short wl") and 550-900 km/s ("long wl") flows are shown in the middle and bottom panels, respectively. The solar wind is symmetric with respect to the ecliptic, with the slow (ecliptic) flow filling the region within $\pm 20 \mathrm{deg}$ from the ecliptic. The sun is in the center at (alpha $=0)$ and (xi=180 deg). The longitudinal asymmetry is explained by the atomic hydrogen distribution in the heliosphere. (c) - The observer (for sky maps shown in (b)) is positioned at 60-deg from the upwind (interstellar wind) direction in the ecliptic plane at 1 AU. (d) - All-sky map (ecliptic coordinates) of the sky brightness at $30.4 \mathrm{~nm}$ due to the glow of the LISM plasma beyond the heliopause (peaks in the upwind direction: longitude $252 \mathrm{deg}$ and latitude $+7 \mathrm{deg}$ ) and pickup ions in the solar wind (peaks in the opposite downwind direction). 
All-sky images of solar wind emissions would map the flow pattern (number density and velocity) of the threedimensional solar wind everywhere in the heliosphere, including over the sun's poles. Most of the solar wind emissions are produced within 10-20 AU (source function) from the sun. The areas in the sky with the high-speed solar wind would stand out in brightness because of the higher velocity-dependent charge-exchange cross sections. In addition, spectral analysis of Doppler-shifted 30.378-nm photons would directly measure the solar wind velocity in a given direction. (A Doppler shift of $0.005 \mathrm{~nm}$ would correspond to a 50-km/s velocity.) Figure 3(b) shows representative calculated all-sky maps of solar wind emissions. ${ }^{18}$ The image clearly shows the boundary between the fast (polar) and slow (ecliptic) solar wind flows for a simple solar wind flow model. In reality, the boundary would be wavy and blurred because of the sun's rotation. We are currently investigating the accuracy of reconstructing this complex boundary in the threedimensional solar wind flow from the multiple images obtained in spectral bins covering possible Doppler-shifts.

We note that the $\mathrm{He}^{+}(2 \mathrm{~s})$ ions produced in charge exchange collisions are in the metastable state (lifetime $1.9 \mathrm{~ms}$ ). These ions would reach the ground state primarily by a two-photon emission process. The continuum radiation produced by this process would dominate continuum diffuse radiation in the heliosphere from $30.4-90 \mathrm{~nm}$ and the averaged stellar radiation field from $32-55 \mathrm{~nm} .^{18}$

\subsection{Galactic emissions}

The galactic EUV radiation field consists of the continuum and line radiation emitted by stars (stellar radiation field) and the radiation emitted by hot interstellar plasmas. The stellar radiation dominates the EUV continuum background at wavelength larger than 20 $\mathrm{nm},{ }^{4,42}$ including the spectral region of interest at $30.4 \mathrm{~nm}$. The stellar radiation field is a continuum with negligible line emissions.

Based on the most complete database obtained by the Extreme Ultraviolet Explorer (EUVE), the total (at least $90 \%$ complete) radiation field is 13.5 phot $\mathrm{cm}^{-2} \mathrm{~s}^{-1} \mathrm{~nm}^{-1}$ around 30 $\mathrm{nm} .^{41}$ If one assumes isotropic background, then this stellar radiation field translates into the $1.3 \times 10^{-2} \mathrm{mR} / \mathrm{nm}$ spectral radiance. ${ }^{18,19}$ The stellar radiation is highly anisotropic, however. Two white dwarfs, G191-2B2 $\left(\lambda=80.6^{\circ}\right.$

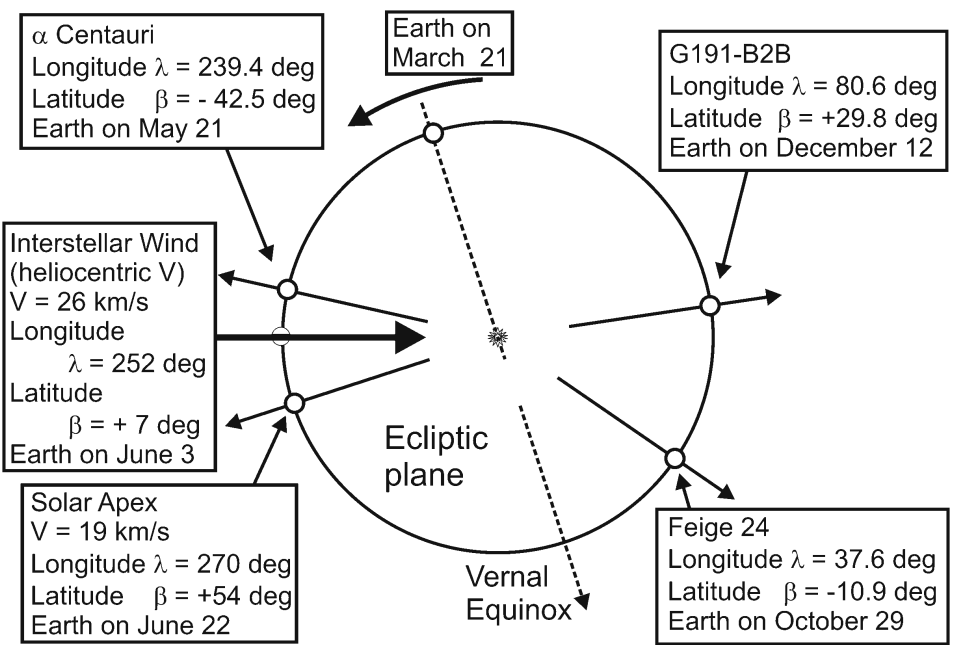

Figure 4. Projection on the ecliptic plane of the most important stellar sources (G191-B2B and Feige 24) of the background radiation at $30.4 \mathrm{~nm}$; $\beta$ and $\lambda$ are ecliptic latitude and longitude, respectively. Also shown are the interstellar wind, solar apex, and closest star, $\alpha$-Centauri. and $\left.\beta=+29.8^{\circ}\right)$ and Feige $24\left(\lambda=37.6^{\circ}\right.$ and $\beta$

$\left.=-10.9^{\circ}\right)$, dominate the background at $30.4 \mathrm{~nm} .{ }^{41}$ For observations in the directions other than these two bright sources (Fig.4), the stellar continuum background would be significantly smaller.

The sun is embedded in a small, several parsec long, and relatively dense $\left(\sim 0.1 \mathrm{~cm}^{-3}\right)$ Local Interstellar Cloud, sometimes also called the local fluff..$^{33-35}$ The cloud is too cold $(7000-8000 \mathrm{~K})$ to emit EUV radiation, but it is surrounded by a region filled with hot $\left(\sim 10^{6} \mathrm{~K}\right)$ and rarefied $\left(\sim 0.005 \mathrm{~cm}^{-3}\right)$ plasmas. This region, called the Local Bubble, may extend up to $200 \mathrm{pc}$ in some directions and even expand into the galactic halo. ${ }^{36}$ The hot plasmas have probably been heated by nearby supernovae with the resulting deviations from thermodynamic equilibrium and cosmic abundances. ${ }^{37}$ We calculate the isotropic EUV background radiation due to the Local Bubble hot plasma using the standard model, ${ }^{38}$ assuming optically thin plasma with the temperature $10^{6} \mathrm{~K}$, emission measure $0.0006 \mathrm{~cm}^{-6} \mathrm{pc}$, absorbing hydrogen slab column density $10^{18} \mathrm{~cm}^{-2}$, and Maxwellian velocity distribution of the plasma ions. ${ }^{18,19}$ Processes at the evaporative boundary of the local cloud ${ }^{39}$ may also contribute to the radiance in EUV; no experimental evidence of such radiation source has been obtained yet and this possibility needs further investigation.

We note that recent measurements (on the Cosmic Hot Interstellar Plasma Spectrometer, or CHIPS, space mission) of diffuse emissions in the wavelength range $9-26.5 \mathrm{~nm}$ suggest that the emissions of hot plasmas in the Local Bubble are 
significantly weaker than by our present concepts predict. ${ }^{40}$ If this important finding is confirmed, then the contribution of the Local Bubble to the radiation background in the heliosphere at $30.4 \mathrm{~nm}$ would be smaller than assumed by us here, with our current estimates thus being conservative.

\subsection{Heliosphere imaging}

Global imaging of the heliosphere at $30.4 \mathrm{~nm}$ will (1) reveal the time-varying three-dimensional properties (number densities and velocities) of the solar wind, including the flow properties in the regions over the sun's poles and on the far side of the sun; (2) map the heliopause and probe the ionization state of the LISM and asymmetry of the magnetic field of the nearby interstellar medium; and (3) measure line emissions of hot plasmas in the Local Bubble. Such a space experiment will contribute to meeting science objectives of two major areas of NASA Science Directorate, study of the Sun-Earth System (former Sun-Earth Connections Division) and Universe (former Astrophysics Division).

The science requirements translate into the following performance characteristics of the instrumentation.

Spectral resolution. Spectral resolution $0.005 \mathrm{~nm}$ would reliably separate major sources (ion glow and solar wind emissions) of radiation at $30.4 \mathrm{~nm}$, unambiguously reveal major details of the solar wind flow, directly measure the flow velocities with a $50-\mathrm{km} / \mathrm{s}$ accuracy, map the heliopause with high precision, and accurately identify emission lines (within the instrument spectral band) of hot plasmas in the Local Bubble.

Spectral band. The proposed instrumentation design (see below) favors rather wide spectral range, almost 4-nm wide, centered on $30.4 \mathrm{~nm}$. (This band can be increased for study of Local Bubble plasmas.)

Angular resolution. The nominal $5^{\circ} \times 5^{\circ}$ angular resolution in heliospheric images would correspond to the characteristic length of $\sim 0.1 \mathrm{AU}$ at a 1-AU distance from the observer. Such angular resolution will reveal numerous details in the solar wind flow pattern in all-sky maps. In addition, one can monitor the position of the fast/slow flow boundary near the heliocentric longitude of the earth by periodically imaging a $100^{\circ}$-long one-dimensional (single-pixel-wide) northsouth swath in the antisolar hemisphere. Large directional gradients of sky brightness are expected over the $15^{\circ}$-long regions across the flow boundaries. ${ }^{18}$ The reconstruction of a complex realistic wavy boundary would require simultaneous images in a number of the $0.005-\mathrm{nm}$ spectral bins obtained across the spectral band corresponding to the entire range of solar wind velocities. To establish the position of the boundary, one would need the measurements in a few points across this transitionary region. Thus, the $5^{\circ} \times 5^{\circ}$ angular resolution will meet the requirements of solar wind imaging.

The heliopause is expected to smoothly vary across the sky ${ }^{18,19,24}$ with the angular scale much larger than $5^{\circ}$. Therefore, the nominal angular resolution (imaging pixel) $5^{\circ} \times 5^{\circ}$ would meet the science requirements of global heliosphere imaging.

Sensitivity. An all-sky image contains $\sim 16005^{\circ} \times 5^{\circ}$ pixels. The heliopause glow would vary slowly with time following the 11-year solar cycle. Obtaining one all-sky image per year during a significant fraction of a solar cycle would allow monitoring the 11-year variations of the heliopause and of the global solar wind flow properties, including - in the case of the latter - the tilt angle of the effective solar magnetic dipole and effective heliographic latitude of the boundary between fast and slow solar wind flows. Emissions of the Local Bubble are not expected to vary at all. Therefore, the minimal instrumentation performance requirement is one all-sky image per year, which corresponds to the exposure of $\sim 20000 \mathrm{sec} /$ pixel $(\sim 5.5 \mathrm{hour} / \mathrm{pixel})$. Typical solar wind emissions would be in the 1-3 $\mathrm{mR}$ range and the expected heliopause glow varies in the $3-15 \mathrm{mR}$ range. ${ }^{18,19}$ We require a $1-\mathrm{mR} /$ bin measurement accuracy $(1 \sigma)$. In other words, the $1-\mathrm{mR} / \mathrm{bin}$ and $10-\mathrm{mR} / \mathrm{bin}$ radiances would be measured with the accuracy of $100 \%$ and $10 \%$, respectively.

A much more ambitious goal to monitor the solar wind changes during the sun's 27-day rotation requires one image per week, which translates into $400 \mathrm{sec} / \mathrm{pixel}$. Since not all observational directions are of equal importance, one can probably ease this requirement to $1000 \mathrm{sec} / \mathrm{pixel}$. The instrument sensitivity of $1000 \mathrm{sec} / \mathrm{pixel}$ would also allow imaging of the $100^{\circ}$-long one-dimensional north-south swath in the antisolar hemisphere by $5^{\circ} \times 5^{\circ}$ pixels in $20000 \mathrm{sec}$, or $\sim 5.5 \mathrm{hr}$. Thus, the fast/slow flow boundary in one direction nearby can be determined a few times a day. The 1000-sec/pixel sensitivity is the ultimate instrument goal. 


\section{ENABLING INSTRUMENTATION}

\subsection{Diffuse radiation spectrometry}

Measurements of weak $(<<1 \mathrm{R})$ diffuse EUV radiation were heretofore almost exclusively the realm of space astrophysics missions studying the emissions from the Local Bubble. The exact processes by which diffuse galactic gas evolves into stars remain a puzzle. While the interstellar gas heating is reasonably well understood, the details of cooling are not that clear. Study of the EUV emissions from the hot $\left(\sim 10^{6} \mathrm{~K}\right)$ gas in the Local Bubble could provide an important insight into the physics of interstellar medium. Interestingly, the results of the recent CHIPS mission have challenged our current concepts. ${ }^{40}$

Main recent astrophysics spectroscopic missions in EUV

$\begin{array}{llcll}\text { Mission } & \text { Band, nm } & \text { Resolution, nm } & \text { Slits } & \text { Launch } \\ \text { EUVE } & 7-70 & 1.7 & 1 & 1992 \\ \text { ALEXIS } & 13-19 & 0.2 & 1 & 1993 \\ \text { EURD } & 35-80 & 0.5 & 1 & 1997 \\ & 50-105 & & & \\ \text { CHIPS } & 9-26 & 0.15 & 6 & 2002\end{array}$

All these recent low-earth orbit missions operate in the bright geocorona. All the spectrographs are single-slit, with the notable exception of CHIPS, where the clever design collects radiation from six slits and diffraction gratings. These missions significantly advanced the state of the art of diffuse radiation spectroscopy. For example, EUV Diffuse Radiation Detector (EURD), launched on the Spanish Minisat-1 and also on Lewis (the spacecraft lost), increased the sensitivity by a factor of $\sim 100$ over similar instruments. ${ }^{41}$

None of the existing instrument designs meets the requirements of heliosphere imaging in EUV. The instruments were also optimized for study of the astrophysical objects with the luxury of exposures up to a Megasecond $\left(10^{6} \mathrm{~s}\right.$ or more than ten days). Our resolution and sensitivity requirements are 2-3 orders of magnitude more demanding. In addition, we require high sensitivity and high spectral resolution simultaneously.

\subsection{Instrument concept}

The main instrumental challenge in detection of the weak diffuse radiation is to maximize the number of photons entering the instrument and to analyze and detect these photons with the utmost efficiency. Noise-free counting of 100 photons provides the $10 \%$ accuracy. However, a (known) detector noise of 1000 counts during the same exposure would reduce the accuracy to $\sim 40 \%$. The minimization of the detector noise is thus an important task.

We propose a quantum advance of instrumentation performance characteristics by fully utilizing the past achievements in the diffuse EUV radiation spectrometry (advanced diffraction gratings, multi-layer coatings, sensitized microchannel plate detectors, anti-coincidence noise-reduction techniques) and by introducing the enabling spectrometer slit design and aperture coding. The time-proven Rowland-type spectrometer is probably the only realistic design that provides the desired spectral resolution and sensitivity under constraints (length, size, weight) of application on a small spacecraft. Availability of multi-layer coatings with high reflectivity at $30.4 \mathrm{~nm}$ makes a Rowland-type normal-incidence spectrometer attractive.

Aperture coding enables the desired breakthrough in the instrumentation performance characteristics. Measurements of the solar wind emissions and the heliopause glow (Fig. 2 and 3a) are characterized by a few bright spectroscopic lines and the dominating signal-independent detector noise. (Most of the detector noise is caused by penetrating radiation.) These are exactly the conditions when application of coding apertures substantially increases the signal and the signalto-noise ratio in measurements of a weak localized signal, such as a spectral line(s), in the presence of the dominating signal-independent detector background noise. ${ }^{42}$ (Coding apertures and conceptually similar techniques are widely used in hard X-ray astrophysics, inertial fusion diagnostics, and time-of-flight molecular beam experiments.) 

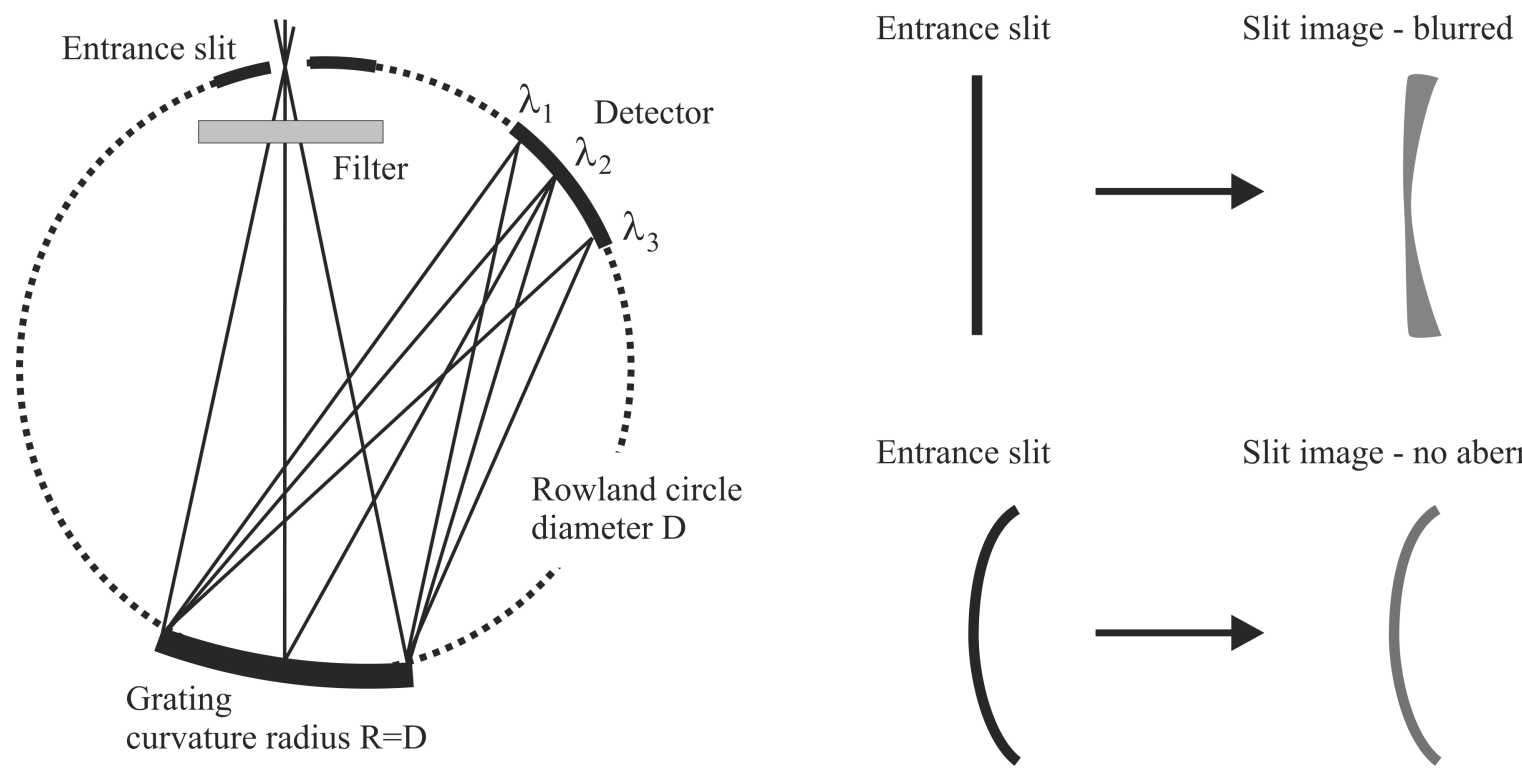

Figure 5. Left - schematic of a Rowland spectrometer for diffuse radiation. Right - the detector image of a straight entrance slit is blurred for tall slits, owing to the inherent Rowland image rotation when used off-plane. Curving the entrance slit restores its inherent resolution off plane. To accommodate a wide entrance field, entrance slits must also be curved towards the grating with twice the curvature of the Rowland circle to remain sharply focused on a flat detector.

Figure 5 shows a concept of a single slit Rowland spectrometer. The spectrometer spectral resolution is determined by the width (for a given spectrometer size) of the entrance slit. Therefore, the slit width cannot be increased without sacrificing spectral resolution. In order to maximize spectrometer throughput and sensitivity, one requires a tall (long) slit. The image of a straight slit on the detector is however curved and blurred as one moves away from the center thus effectively limiting the throughput. This effect is caused by the inherent Rowland image rotation when used off-plane. When combined with the usual straight (vertical) entrance slit, a Rowland mount sacrifices spectral resolving power as the slit is made taller.

We investigated various spectrometer designs that maximize the working zone while preserving the desired spectral resolution. ${ }^{25,26}$ In our design we require to replace the single slit with a large number $(>1000)$ of slits covering a large area. Consequently, the spectrometer working zone has to be unusually large. Multiple spectral images overlap on the photon-counting detector and the original spectrum is extracted after deconvolution of the scrambled image. The normal-incidence Rowland mount, with the slit group located around off-plane angle $\beta=0$ and the detector located at the first order diffraction angle, provides optimum performance for the intended application. ${ }^{26}$ Curving the entrance slits (Fig. 5; right) restores inherent resolution off-plane, while additional curving towards the grating with twice the curvature of the Rowland circle keeps the image sharply focused on a flat detector.

The details of the spectrometer design are provided elsewhere. ${ }^{26}$ In addition, the flight instrument design would maximize efficiency of the diffraction grating through optimization of their multi-layer coatings; maximize suppression of the stray photon background by optimization of the detector and grating coatings (and possible filter) properties; and minimize detector noise by further advance of the highly-successful anti-coincidence technique employed on EURD. ${ }^{41}$

\subsection{Aperture coding}

We replace the conventional single slit of the spectrometer by an aperture consisting of two $\mathrm{N}$-sets of slit elements which could be either open ("hole"), i.e. transmitting the incident radiation, or opaque. The aperture is described mathematically as a binary sequence, with 1's corresponding to a slit (transmitting radiation) and 0's corresponding to 
opaque elements. The number of open elements, $n$, is usually $n \approx N / 2$. Each open slit element would produce an identical but shifted spectrum on the detector sensitive surface. Thus, the $n$ identical spectra overlap (multiplex) on the detector. The resulting (scrambled) image can be unscrambled by postprocessing and the original spectrum restored if one knows the exact slit positions at the aperture. The best sequence of the coding slits is provided by pseudorandom sequences, which minimizes deconvolution errors and artifacts. ${ }^{42}$ The number of slits is almost exactly equal to the number of the opaque intervals that block radiation passage. The width of the each opaque (blocking) element exactly equals the width of the slit.

The theory of coding aperture and its applications for imaging of physical objects in space are well understood. ${ }^{42}$ Briefly, let us assume that

1) the object (the spectrum to be measured) is an $\mathrm{N}$-element vector $\mathbf{o}$ with elements $\mathrm{o}_{\mathrm{i}}, \mathrm{i}=0, \ldots, \mathrm{N}-1$;

2) the aperture is a binary sequence $\mathbf{b}$ of the length $2 \mathrm{~N}$ (elements $b_{i}, i=0, \ldots, 2 \mathrm{~N}-1$ ) consisting of two consecutive basic sequences a with elements $a_{i}, i=0, \ldots, N-1$; and $b_{j}=b_{j+N}$ for $N \leq j<2 N$;

3) the registered image is a vector $\mathbf{p}$ with elements $\mathrm{p}_{\mathrm{i}}, \mathrm{i}=0, \ldots, \mathrm{N}-1$.

The registered image is then

$$
p_{k}=\sum_{i=0}^{N-1} o_{i} b_{i+k}+u_{k}
$$

where the vector $\mathbf{u}$ (with elements $\mathrm{u}_{\mathrm{i}}, \mathrm{i}=0, \ldots, \mathrm{N}-1$ ) is the signal-independent noise counts. A cyclic matrix $\mathbf{A}$ (size $\mathrm{N}$ by N) can then be constructed from the basic sequence $\mathbf{a}$ in such a way that the first row of $\mathbf{A}$ is the basic sequence $\mathbf{a}$ and each following row is obtained from the previous one by cyclic shift of one step, i.e. $A_{i j}=a_{i+j-1}$. The registered image is

$$
\mathbf{p}=\mathbf{A} \mathbf{o}+\mathbf{u}
$$

Reconstruction of the original object $\mathbf{o}$ consists of convolution with the postprocessing array $\mathbf{G}$

$$
\mathbf{G} \mathbf{p}=(\mathbf{G} * \mathbf{A}) \mathbf{o}+\mathbf{G} \mathbf{u}
$$

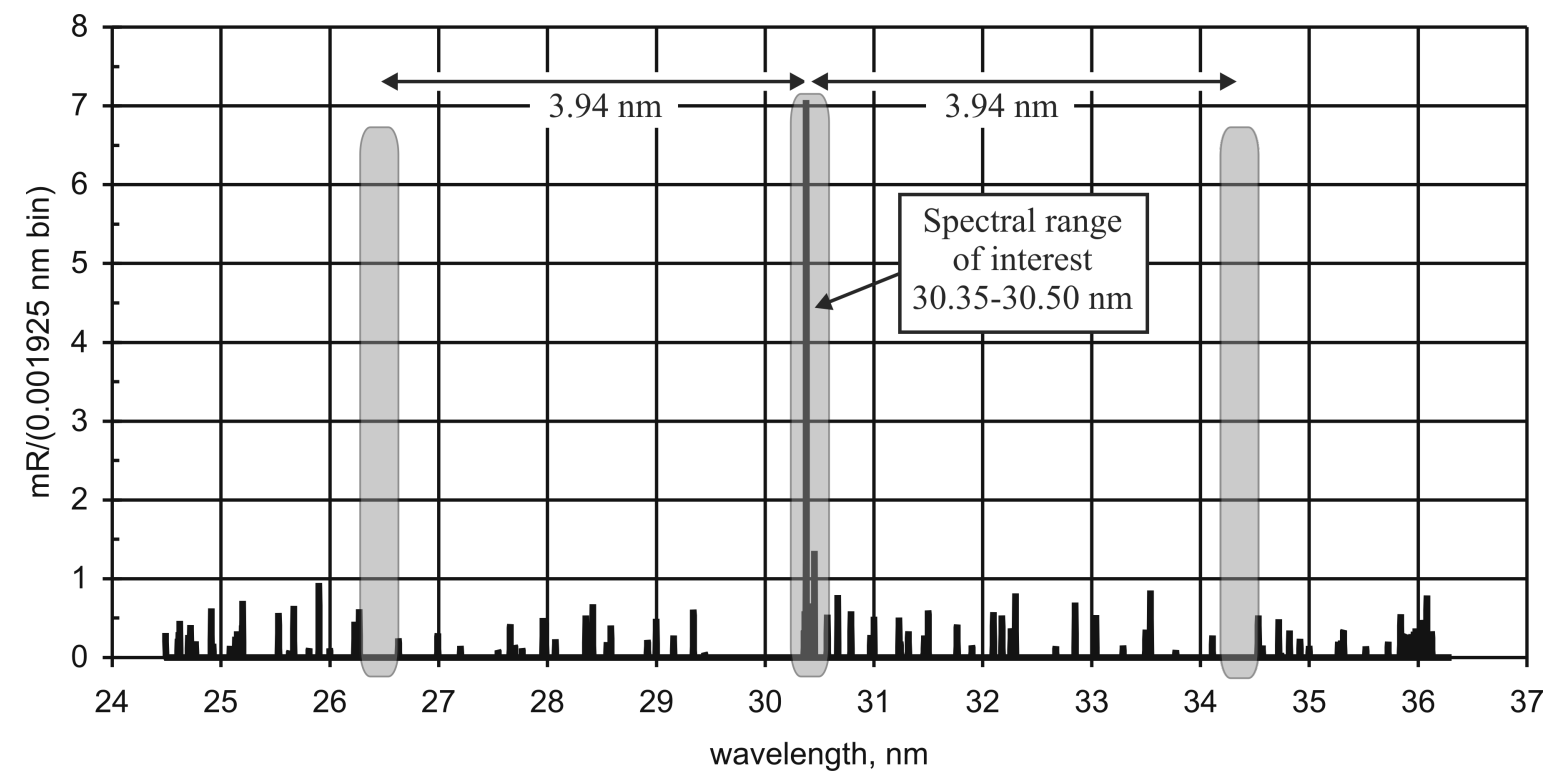

Figure 6. Expected spectral radiance from 26-37 nm from the glow of interstellar plasma and solar wind pick up ions, solar wind emissions, and emissions of hot plasmas in the Local Bubble. The spectral range of interest for heliopause imaging and mapping the solar wind flow is from 30.35-30.50 nm (highlighted in the center) - see also Fig.2. Short spectral lines ("grass") throughout the range originate in the Local Bubble. Recent CHIPS measurements suggest that these line emissions may be smaller. ${ }^{40}$ Two side highlighted areas are shifted $3.94 \mathrm{~nm}$ from the 30.35-30.50 nm range and do not contain interstellar lines. Therefore, a coded-aperture instrument with the 3.94-nm spectral range would avoid artifacts caused by partial coding. 
The array $\mathbf{G}$ is also a cyclic matrix constructed from the basic sequence $g_{i}, i=0, \ldots, 2 N-1\left(\right.$ and $g_{j}=g_{j+N}$ for $\left.N \leq j<2 N\right)$ in a way similar to that of matrix $\mathbf{A}$. Then, if $\mathbf{G} \mathbf{A}=\mathbf{I}$, where $\mathbf{I}$ is the identity matrix, then

$$
o_{k}=\sum_{i=0}^{N-1} p_{i} g_{i+k}-\sum_{i=0}^{N-1} u_{i} g_{i+k} .
$$

The quality of an imaging system can be quantitatively described in terms of the point-spread function (PSF). PSF is a reconstructed image of the $\delta$-function object, and in our case, PSF is equal to the correlation of the aperture transmission function with the postprocessing function. If PSF is not equal to the $\delta$-function, then the image reconstruction with the correlation postprocessing is prone to artifacts or inherent inaccuracy, as it is sometimes called. The so-called pseudorandom binary sequences consisting of 1's and 0's provide the desired perfect autocorrelation function. A pseudorandom binary sequence is generated deterministically but the distribution of 1's and 0's in the sequence exhibits statistical properties of random sequence.

The procedure for constructing pseudo-random sequences is well known. ${ }^{42-44}$ A primitive polynomial $\mathrm{h}(\mathrm{x})$ of degree $\mathrm{m}$ determines the pseudorandom sequence of length $\mathrm{N}=2^{\mathrm{m}}-1$. (The primitive polynomial determines the exact feedback shift register generating the binary sequence.) The first 16 primitive polynomials are

Polynomial $\mathrm{h}(\mathrm{x}) \quad$ Polynomial degree $\mathrm{m}$

$\begin{array}{ll}x+1 & 1 \\ x^{2}+x+1 & 2 \\ x^{3}+x+1 & 3 \\ x^{4}+x+1 & 4 \\ x^{5}+x^{2}+1 & 5 \\ x^{6}+x+1 & 6 \\ x^{7}+x+1 & 7 \\ x^{8}+x^{6}+x^{5}+x+1 & 8 \\ x^{9}+x^{4}+1 & 9 \\ x^{10}+x^{3}+1 & 10 \\ x^{11}+x^{2}+1 & 11 \\ x^{12}+x^{7}+x^{4}+x^{3}+1 & 12 \\ x^{13}+x^{4}+x^{3}+x+1 & 13 \\ x^{14}+x^{12}+x^{11}+x+1 & 14 \\ x^{15}+x+1 & 15 \\ x^{16}+x^{5}+x^{3}+x^{2}+1 & 16\end{array}$

Sequence length $\mathrm{N}$ (number of elements in aperture basic sequence)

$\begin{array}{lll}2 & 1 & 0 \text { or } 1 \\ 3 & 7 & 1 \text { or } 2 \\ 4 & 15 & 3 \text { or } 4 \\ 5 & 31 & 7 \text { or } 8 \\ 6 & 63 & 15 \text { or } 16 \\ 7 & 127 & 31 \text { or } 32 \\ 8 & 255 & 63 \text { or } 64 \\ 10 & 511 & 127 \text { or } 128 \\ 1 & 1023 & 255 \text { or } 256 \\ 13 & 2047 & 511 \text { or } 512 \\ 14 & 4095 & 1023 \text { or } 1024 \\ 15 & 8191 & 2047 \text { or } 2048 \\ 16 & 16383 & 4095 \text { or } 4096 \\ & 32767 & 8191 \text { or } 8192 \\ 65535 & 16383 \text { or } 16384\end{array}$

Number $n$ of 1 's
(open slit elements)
in aperture basic sequence
0 or 1
1 or 2
3 or 4
7 or 8
15 or 16
31 or 32
63 or 64
127 or 128
255 or 256
511 or 512
1023 or 1024
2047 or 2048
4095 or 4096
8191 or 8192
16383 or 16384
32767 or 32768

Number n of 1's (open slit elements) ensic sequence

Consider a pseudo-random sequence a, with the number of elements N. The number of openings ("holes" allowing the incident radiation to enter the spectrometer) or 1 's in the sequence is $n$, and $n \approx N / 2$. The deconvolution sequence $g$ is then

and

$$
\begin{array}{rlll}
g_{i}=1 / n, & \text { if } & a_{i}=1 \\
-1 / n, & \text { if } & a_{i}=0
\end{array}
$$

$$
\begin{aligned}
\sum_{i=0}^{N-1} g_{i} a_{i-k} & =1, & \text { if } & k=0 \\
& =0, & \text { if } & k \neq 0
\end{aligned}
$$




\subsection{Partial coding and instrument spectral band}

Figure 6 shows the expected radiance around $30.4 \mathrm{~nm}$. The lines of interest (solar wind emissions and glow of the LISM plasma and pickup ions) stand out near $30.4 \mathrm{~nm}$ (Figures 2 and 3a). The numerous other small lines ("grass") are the emissions of hot interstellar plasma ions in the Local Bubble, as predicted by the standard model of hot plasma emission. ${ }^{19,38}$ The continuum background is negligible. ${ }^{18,19}$

Aperture coding may produce the artifacts due to the signal that is not projected on the detector through all of the aperture slits ("partial coding"). ${ }^{42}$ Consider an instrument with the nominal $\Delta \lambda$-wide spectral band (Fig. 7). Only the radiation entering through the center of the aperture $\left(\mathrm{k}_{3}\right)$ maps on the detector ( $\mathrm{N}$ bins) the exact spectral range of interest $\Delta \lambda$. All other aperture elements (slits) map on the detector 1) parts of the spectral range $\Delta \lambda$ (black) and 2) additional emissions (gray) outside of the spectral range $\Delta \lambda$. Each detector element is thus exposed exactly to all spectral bins of the spectral range of interest $\Delta \lambda$ (full coding). For example, consider the leftmost detector element. It captures the leftmost spectral element of the range $\Delta \lambda$ from the entrance slit $k_{3}$, the central spectral element from the entrance slit $\mathrm{k}_{2}$, and the rightmost spectral element from the entrance slit $k_{1}$.

Spectral bins outside the spectral range $\Delta \lambda$ reach only parts of the detector (partial coding) and would produce artifact peaks in reconstructed spectra. In the reconstructed (from the measurement) spectrum, a spectral line at a wavelength $\lambda_{0}$ outside the instrument range would show up (with somewhat reduced intensity) within the instrument range at the position corresponding to the wavelength $\left(\lambda_{0}-\Delta \lambda\right)$ or $\left(\lambda_{0}+\Delta \lambda\right)$. The spectral lines are thus "wrapped around" the nominal spectral band. ${ }^{42}$

Partial coding could be substantially reduced by eliminating emissions outside the spectral band $\Delta \lambda$ by a narrow grating reflection curve and/or by the filter at the entrance. The maximized reflection at $30.4 \mathrm{~nm}$ could exceed 20\% ${ }^{45-47}$ A narrow reflection band, such as 4-nm FWHM on IMAGE ${ }^{47}$ necessarily reduces the peak reflectivity, which is not desirable.

Our approach (Fig. 7) does not require the narrow spectral range. (The narrow transmission band would be helpful, however, in reducing detector count rate and improving weak signal extraction.) We suppress the partial coding artifacts by selecting the instrument range 3.94-nm wide. In that case, no wrap-around lines would appear in the $30.35-30.50 \mathrm{~nm}$ range, because no interstellar lines are expected around $(30.4 \pm 3.94) \mathrm{nm}$ (Fig.6). Actually, the range from $30.21 \mathrm{~nm}$ to $30.57 \mathrm{~nm}$ would be clear from the artifact interstellar lines.

\subsection{Instrument}

The design and performance characteristics of the Rowland mount spectrometer using the curved slit principle to achieve a large entendue without significant resolution penalty is presented elsewhere. ${ }^{25,26} \mathrm{We}$ demonstrate here the performance of the proposed instrumental concept based on aperture coding with the following parameters:

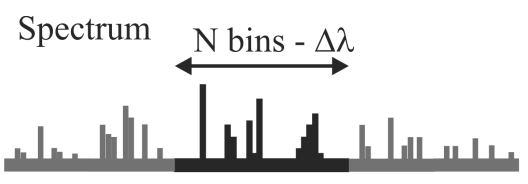

Aperture basic sequence - $\mathrm{N}$ elements $\stackrel{\longleftrightarrow}{\longleftrightarrow \|}$

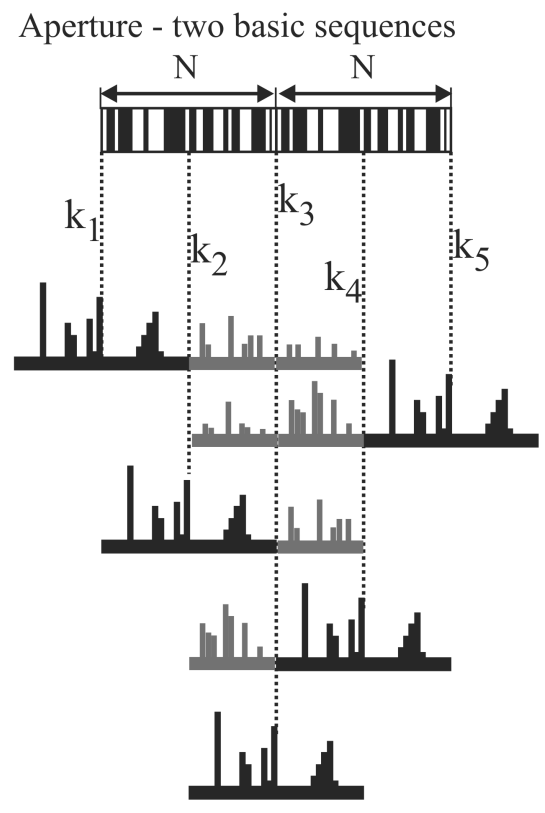

Detector

Figure 7. Top - the instrument is designed to detect the spectrum $\Delta \lambda$ of $\mathrm{N}$ bins (black). The emissions outside of this spectral range (gray) will contribute to artifacts caused by partial coding. The aperture basic sequence also consists of $\mathrm{N}$ elements. Bottom - two aperture basic sequences comprise the aperture. Only the radiation entering through the center of the aperture $\left(\mathrm{k}_{3}\right)$ maps on the detector ( $\mathrm{N}$ bins) the exact spectral range of interest $\Delta \lambda$. All other aperture elements (slits) map on the detector 1) parts of the spectral range $\Delta \lambda$ (black) and 2) emissions (gray) outside of the spectral range $\Delta \lambda$. Each detector element is exposed exactly to all spectral bins of the spectral range of interest $\Delta \lambda$ (full coding). Spectral bins outside this spectral range $\Delta \lambda$ reach only parts of the detector (partial coding). 
Coding aperture basic sequence -2047 elements

Aperture - 4094 elements

Aperture element width $-0.002 \mathrm{~cm}$ (20 micron)

Aperture element length $-6 \mathrm{~cm}$

Aperture area $-8.2 \times 6.0 \mathrm{~cm}^{2}$

Field-of-view $-2.75^{\circ} \times 5^{\circ}$

Spectral resolution $-0.001925 \mathrm{~nm}$

Spectral range $-3.94 \mathrm{~nm}(2047 \times 0.001925 \mathrm{~nm})$

Detector noise count rate -5.3 count/s

Instrument total detection efficiency -0.03

The instrument count rate is 14.3 count/s, which includes 5.3 count/s detector noise (intrinsic noise and counts due to penetrating radiation). Figure 8 (top) shows the original spectrum to be detected. The large peak corresponds the ion glow and two peaks between 30.4 and $30.5 \mathrm{~nm}$ are the Dopplershifted solar wind emissions. The middle panel of Fig.8 shows the simulated reconstructed image after accumulation time of $10000 \mathrm{~s}(\sim 2.8 \mathrm{hr})$. One can clearly see the glow peak and the larger solar wind emission peak, thus validating the proposed instrument concept and meeting the minimal instrument requirements described in Section 2 of this article. The overall efficiency of photon reflection (diffraction grating) and detection (by a position-sensitive detector based on microchannel plates) is assumed 0.03 . This is a conservative estimate of instrument efficiency.

Two main sources of noise contribute here: detector noise and the unavoidable Poissonian noise associated with the low count rates. About $60 \%$ of the detector count rate is produced by line emissions outside the spectral range of interest $(30.3-30.5 \mathrm{~nm})$. So, if grating reflection and detector efficiency are optimized to suppress the out of range radiation, the signal extraction will be further improved. Increase of the accumulation time will also improve signal extraction by reducing the Poissonian noise. Figure 8 (bottom) shows the simulated detector output accumulated by a single-slit spectrometer under similar conditions. Clearly no detection is possible without multiple aperture slits.

\section{SPACE MISSION}

Measurement of weak emissions requires minimal detector noise. Therefore, the spacecraft must be placed in an orbit outside the magnetosphere of the Earth and outside the geocorona. Spacecraft in high-apogee Earth orbits satisfy such re-
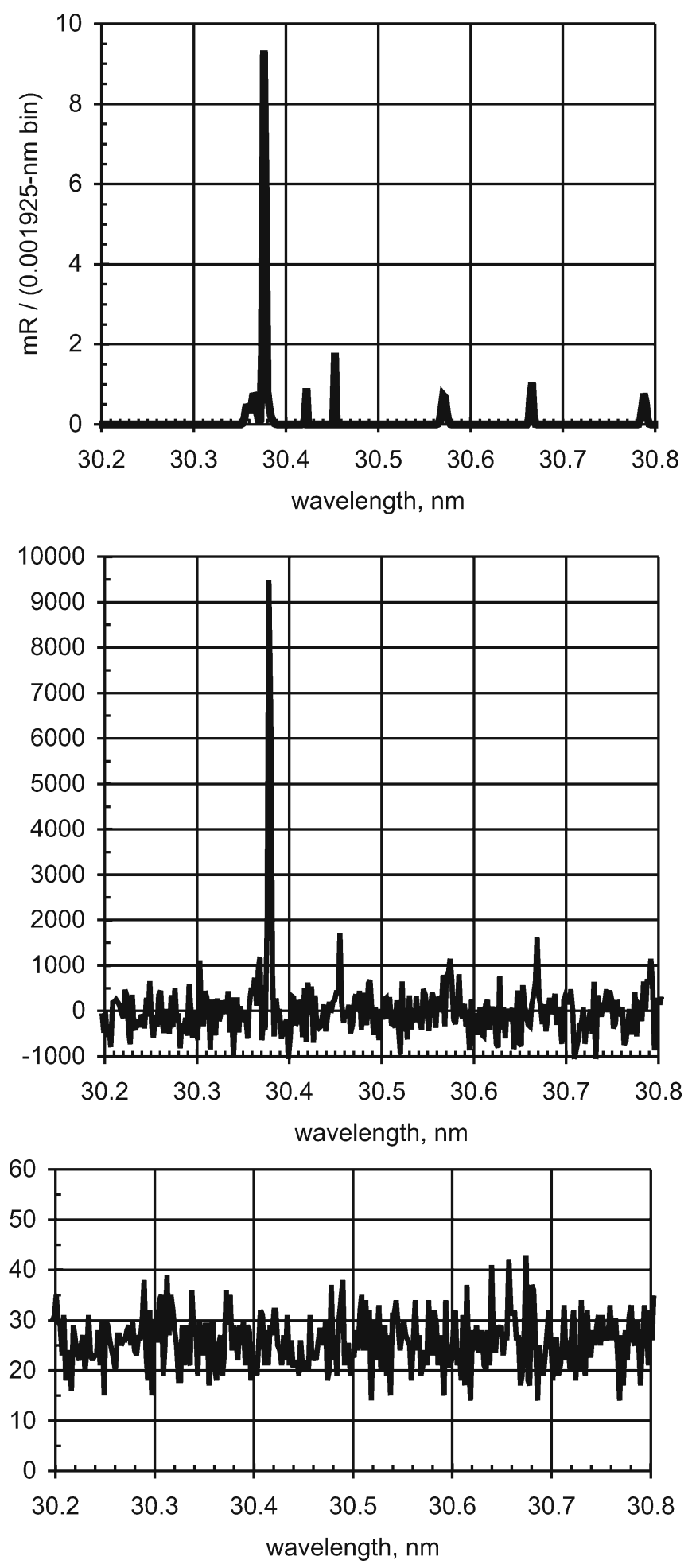

Figure 8. Top - original spectrum to be detected; middle - reconstructed spectrum by the multi-slit coded-aperture spectrometer; bottom - detector output by a single-slit spectrometer under similar conditions. 


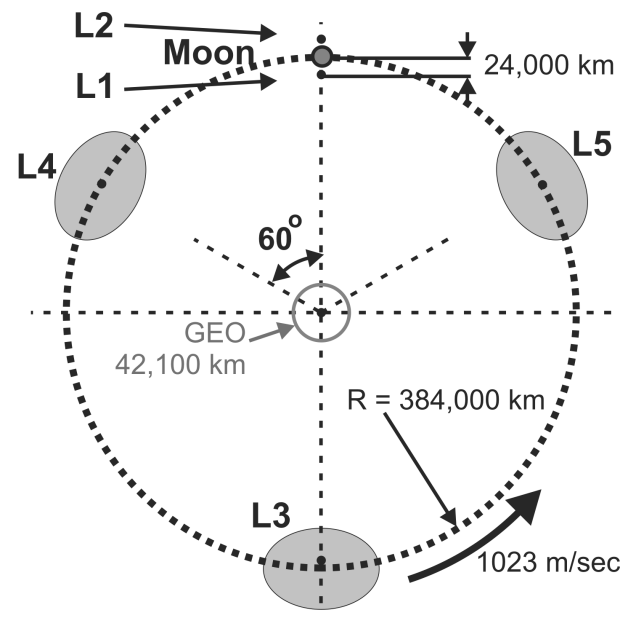

Figure 9. Earth-Moon libration points L4, L5, and L3 offer excellent locations (gray areas) for deploying a spacecraft to conduct heliosphere imaging in EUV. A geostationary orbit (GEO) is shown for comparison. quirements during part of the orbit. The observations however would need to interrupt during the perigee passes which also exposes spacecraft to high fluxes of energetic charged particles in the radiation belts. The recently selected NASA's Interstellar Boundary Explorer ${ }^{13}$ has the mission requirements conceptually similar to those of heliosphere imaging in EUV: the spacecraft maps the heliosphere in weak ENA fluxes. The IBEX spacecraft will be deployed in a high-apogee orbit using a small launcher (Pegasus) and the mission will be accomplished within the cost limits of the Small Explorer (SMEX) program.

An interplanetary mission would provide the perfectly quiet observational conditions for heliosphere EUV imaging but it requires high delta-V launch capabilities for deployment and large antennas for communications with the spacecraft (and expensive operations). These requirements consequently drive the cost up, making a space mission to image the heliosphere in EUV less competitive for limited funds in NASA.

We propose a new mission concept that combines advantages of quiet environmental conditions of an interplanetary mission, relatively low delta- $\mathrm{V}$ requirements of a high-apogee Earth orbiting mission, straightforward spacecraft design, and simplicity and low cost of mission operations and control. While such a mission is still more costly than what can be realistically accomplished under the SMEX program, it would fit within the cost limits of a Medium Explorer (MIDEX) mission with the cost significantly smaller than the MIDEX cap.

We propose to deploy a spacecraft in a libration point, L4, L5, or L3, of the Earth-Moon system (Fig. 9). In the rotating Earth-Moon reference frame, there are five points - L1, L2, L3, L4, L5 - of gravitational equilibrium (under the assumption of vanishing disturbing sun's gravity). All points are in the plane of the Moon's orbit around the Earth. Points L4 and L5 form equilateral triangles with the Earth and the Moon as shown in Fig. 9 and they are inherently stable - a body (spacecraft) placed in L4 or L5 would stay in these points (in the Earth-Moon rotating reference frame) indefinitely. The sun's gravity disturbs this ideal case, so the spacecraft would move around these libration points in an open halo orbit. What is important for our purposes is that the spacecraft would stay in the approximate vicinity of a libration point outside the magnetosphere and would require minimal delta- $\mathrm{V}$ (several $\mathrm{m} / \mathrm{s}$ ) for station keeping during the duration of a mission lasting several years.

Spacecraft deployment to L4 or L5 can be efficiently achieved using lunar gravity assist. The deployment from a lowearth parking orbit would require delta-V less than $3.3 \mathrm{~km} / \mathrm{s}$, including about $3.1 \mathrm{~km} / \mathrm{s}$ for the initial boost from the parking orbit, $0.1 \mathrm{~km} / \mathrm{s}$ for midcourse maneuvers with a very generous margin, and $0.1 \mathrm{~km} / \mathrm{s}$ for phasing into the libration point station. Note that this delta- $\mathrm{V}$ is significantly smaller than the velocity requirements for placing a spacecraft into a geostationary orbit (GEO), $4.2 \mathrm{~km} / \mathrm{s}$ for a Cape Canaveral launched spacecraft. Libration points L4 and L5 attracted space visionaries and enthusiasts (e.g., "L5 Society" of 1970s) for many years as possible locations for space stations and colonies, but they have never been used for a scientific spacecraft.

The conditions at L4 or L5 are perfect for a mission to image the heliosphere in EUV. The spacecraft would be at the distance $\sim 384,000 \mathrm{~km}$ from the Earth, well beyond the geocorona and the magnetosphere, with uninterrupted observations for almost $100 \%$ of the time. The absence of eclipses would significantly simplify spacecraft design, especially its thermal control and electric power subsystems. Minimal environmental torques (the largest environmental torque would be produced by solar light pressure) minimize requirements to the spacecraft attitude control system. Actually, solar radiation pressure can be used for spacecraft attitude control. A relatively short (compared to interplanetary spacecraft) distance to the spacecraft makes communications easy. A small 5-W spacecraft transmitter can easily provide a 10-kb/s communication link for a $1.5 \mathrm{~m}$ ground antenna. In addition, the spacecraft would slowly move in the sky, making its tracking easy and allowing long uninterrupted access (up to 10 hours) from the same location. 
Mapping of the sky can be conveniently achieved by a three-axis stabilized spacecraft. Alternatively, a spinning spacecraft can also provide global imaging of the heliosphere. ${ }^{12,13}$ A spinning spacecraft with the spin axis pointed at the Sun also offers additional simplicity of spacecraft design and operations. By precessing the spacecraft Sun-pointed axis as the Earth moves around the Sun, the full sky coverage is achieved in 6 months by a single pixel instrument pointed normally to the spin axis. ${ }^{12}$ Such an arrangement, however, would cover various regions of the sky with highly nonuniform efficiency (dwelling time, or duty cycle).

\section{ACKNOWLEDGEMENT}

This work is supported in part by a NASA grant.

\section{REFERENCES}

1. L. Davis, Jr., "Interplanetary magnetic fields and cosmic rays," Phys. Rev., 100, 1440-1444, 1955.

2. E.N. Parker, Interplanetary Dynamical Processes, Wiley-Interscience, New York, 1963.

3. A.J. Dessler, "Solar wind and interplanetary magnetic field," Rev. Geophys., 5, 1-41, 1967.

4. W.I. Axford, "The interaction of the solar wind with the interstellar medium," Solar Wind, NASA Spec. Publ., SP308, 609-660, 1972.

5. V.B. Baranov, "Gasdynamics of the solar wind interaction with the interstellar medium," Space Sci. Rev., 52, 89$120,1990$.

6. S.T. Suess, S.T., "The heliopause," Rev. Geophys., 28, 97-115, 1990.

7. H.J. Fahr and H. Fichtner, "Physical reasons and consequences of a three-dimensionally structured heliosphere," Space Sci. Rev., 58, 193-258, 1991.

8. G.P. Zank, "Interaction of the solar wind with the local interstellar medium: A theoretical perspective," Space Sci. Rev., 89, 1-275, 1999.

9. S.M. Krimigis, R.B. Decker, M.E. Hill, T.P. Armstrong, G. Gloeckler, D.C. Hamilton, L.J. Lanzreotti, and E.C. Roelof, "Voyager 1 exited the solar wind at a distance of $~ 85$ AU from the Sun," Nature, 426, 45-48, 2003.

10. F.B. McDonald, E.C. Stone, A.C. Cummings, B. Heikkila, N. Lal, and W.R. Webber, "Enhancements of energetic particles near the heliospheric termination shock," Nature, 426, 48-51, 2003.

11. S. Jaeger and H.J. Fahr, "The heliospheric plasma tail under influence of charge exchange processes with interstellar H-atoms," Sol. Phys., 178, 631-656, 1998.

12. M. Gruntman, E.C. Roelof, D.G. Mitchell, H.J. Fahr, H.O. Funsten, and D. J. McComas, "Energetic neutral atom imaging of the heliospheric boundary region,” J. Geophys. Res., 106, 15767-15781, 2001.

13. D. McComas, F. Allegrini, P. Bochsler, M. Bzowski, M. Collier, H. Fahr, H. Fichtner, P. Frisch, H. Funsten, S. Fuselier, G. Gloeckler, M. Gruntman, V. Izmodenov, P. Knappenberger, M. Lee, S. Livi, D. Mitchell, E. Moebius, T. Moore, D. Reisenfeld, E. Roelof, N. Schwadron, M. Wieser, M. Witte, P. Wurz, and G. Zank, "The Interstellar Boundary Explorer (IBEX)," Physics of the Outer Heliosphere, AIP Conf. Proc., Vol. 719, 162-181, Melville, NY: American Institute of Physics, 2004.

14. M. Neugebauer, "The three-dimensional solar wind at solar activity minimum," Rev. Geophys., 37, 107-126, 1999.

15. R. Lallement, E. Quemerais, J.L. Bertaux, S. Ferron, D. Koutroumpa, and R. Pellinen, "Deflection of the interstellar neutral hydrogen flow across the heliospheric interface," Science, 307, 1447-1449, 2005.

16. M. Gruntman, "Instrumentation for interstellar exploration," Adv. Space Res., 34, 204-212, 2004.

17. M. Gruntman, "Energetic neutral atom imaging of space plasmas," Rev. Sci. Instrum., 68, 3617-3656, 1997.

18. M. Gruntman, "Imaging the three-dimensional solar wind," J. Geophys. Res., 106, 8205-8216, 2001.

19. M. Gruntman, "Mapping the heliopause in EUV," The Outer Heliosphere: The Next Frontiers, Pergamon, 263-271, 2001.

20. M. Gruntman and V. B. Leonas, "Neutral solar wind: possibilities of experimental investigation," Report (Preprint) 825, Space Research Institute (IKI), Academy of Sciences, Moscow, 1983.

21. M. Gruntman, V.B. Leonas, and S. Grzedzielski, "Neutral solar wind experiment," Physics of the Outer Heliosphere, 355-358, Pergamon Press, New York, , 1990.

22. C.J. Pollock, K. Asamura, J.Baldonado, M.M. Balkey, P. Barker, J.L. Burch, E.J. Korpela, J. Cravens, G. Dirks, M.-C. Fok, H.O. Funsten, M. Grande, M. Gruntman, J. Hanley, J.-M. Jahn, M. Jenkins, M. Lampton, M. Marckwordt, D.J. McComas, T. Mukai, G. Penegor, S. Pope, S. Ritzau, M.L. Schattenburg, E. Scime, R..; Skoug, W. 
Spurgeon, T. Stecklein, S. Storms, C. Urdiales, P. Valek, J.T.M. van Beek, S.E. Weidner, M. Wuest, M.K. Young, and C. Zinsmeyer, "Medium energy neutral atom (MENA) imager for the IMAGE mission," Space Sci. Rev., 91, 113-154, 2000.

23. M. Gruntman, and H.J. Fahr, "Access to the heliospheric boundary: EUV-echoes from the heliopause," Geophys. Res. Lett., 25, 1261-1264, 1998.

24. M. Gruntman and H.J. Fahr, "Heliopause imaging in EUV: Oxygen $\mathrm{O}^{+}$ion 83.4-nm resonance line emission," J. Geophys. Res., 105, 5189-5200, 2000.

25. M. Lampton, J. Edelstein, T. Miller, and M. Gruntman, “A high-throughput, high-resolution spectrometer for mapping the heliopause and 3-D Solar Wind using $\mathrm{He}^{+}$30.4nm,” Eos Trans. AGU, 85(47), Fall Meeting Suppl., Abstract SH31A-1181, 2004.

26. M. Lampton, J. Edelstein, T. Miller, and M. Gruntman, "An EUV spectrometer for mapping the heliopause and solar wind," UV, X-Ray, and Gamma-Ray Space Instrumentation for Astronomy XIV, ed. O.H.W. Siegmund, Proc. SPIE 5898, to be published, 2005.

27. E. Moebius, D. Hovestadt, B. Klecker, M. Scholer, and G. Gloeckler, "Direct observation of He pickup ions of interstellar origin in the solar wind," Nature, 318, 426-429, 1985.

28. G. Gloeckler, "The abundance of atomic ${ }^{1} \mathrm{H},{ }^{4} \mathrm{He}$, and ${ }^{3} \mathrm{He}$ in the local interstellar cloud from pickup ion observation on Ulysses," Space Sci. Rev., 78, 335-346, 1996.

29. E. Moebius, "The local interstellar medium viewed through pickup ions, recent results and future perspectives," Space Sci. Rev., 78, 375-386, 1996.

30. M. Gruntman and V. Izmodenov, "Mass transport in the heliosphere by energetic neutral atoms," J. Geophys. Res., 109, A12108, doi: 10.1029/2004JA010727, 2004.

31. J. Vallerga, "The stellar extreme-ultraviolet radiation field," Astrophys. J., 497, 921-927, 1988.

32. K.-P. Cheng and F.C. Bruhweiler, "Ionization processes in the local interstellar medium: Effects of the hot coronal substrate,” Astrophys. J., 364, 573-581, 1990.

33. R. Lallement, R. Ferlet, A.M. Lagrange, M. Lemoine, and A. Vidal-Madjar, "Local interstellar cloud structure from HST-GHRS," Astron. Astrophys., 304, 461-474, 1995.

34. P.C. Frisch, "Characteristics of nearby interstellar medium," Space Sci. Rev., 72, 499-592, 1995.

35. J.L. Linsky, S. Redfield, B.E. Wood, and N. Piskunov, "The three-dimensional structure of the warm local interstellar medium. I. Methodology,” Astrophys. J., 528, 756-766, 2000.

36. B.Y. Welsh, D.M. Sfeir, M.M. Sirk, and R. Lallement, "EUV mapping of the local interstellar medium: the Local Chimney revealed?" Astron. Astrophys., 352, 308-316, 1999.

37. D.P. Cox and R.J. Reynolds, “The local interstellar medium,” Annu. Rev. Astron. Astrophys., 25, 303-344, 1987.

38. M. Landini and B.C. Monsignori Fossi, "The X-UV spectrum of thin plasmas," Astron. Astrophys. Suppl. Ser., 82, 229-260, 1990.

39. J.D. Slavin, "Consequences of a conductive boundary on the local cloud. I. No dust," Astrophys. J., 346, 718-7272, 1989.

40. M. Hurwitz, T.P. Sasseen, M.M. Sirk, "Observations of diffuse extreme-ultraviolet emissions with the Cosmic Hot Interstellar Plasma Spectrometer (CHIPS),” Astrophys. J., 623, 911-916, 2005.

41. S. Bowyer, J. Edelstein, and M. Lampton, "Very high sensitivity extreme ultraviolet spectrometer for diffuse radiation," Astrophys. J., 485, 523-532, 1997.

42. M. Gruntman, "Coded-aperture technique for magnetosphere imaging: advantages and limitations," Instrumentation for Magnetospheric Imagery II, ed. S. Chakrabarti, Proc. SPIE 2008, 58-73, 1993.

43. S.W. Golomb, Digital communications with space applications, Prentice Hall, Englewood Cliffs, NJ, 1964.

44. F.J. MacWilliams and N.J.A. Sloane, "Pseudo-random sequences and arrays," Proc.IEEE, 64(12), 1715-1729, 1976.

45. M. Nakamura, T. Yamamoto, K. Tsuruda, Y. Saito, K. Yamashita, A. Furuzawa, T. Ogawa, and S. Chakrabarti, "Interplanetary He II extreme-ultraviolet observation on PLANET-B," Optical Engineering, 32, 3033-3037, 1993.

46. B.C. Bush, D.M. Cotton, and S. Chakrabarti, "Coronal ultraviolet Berkeley spectrometer," Optical Engineering, 32, 3047-3053, 1993.

47. B.R. Sandel, A.L. Broadfoot, C.C. Curtis, R.A. King, T.C. Stone, R.H. Hill, J. Chen, O.H.W. Siegmund, R. Raffanti, D.D. Allred, R.S. Turley, and D.L. Gallagher, "The extreme ultraviolet imager investigation fir the IMAGE mission," Space Sci. Rev., 91, 197-242, 2000. 